\title{
FLUIDIZATION OF GAS-SOLID IN ATMOSPHERIC BUBBLING FLUIDIZED BED COMBUSTOR
}

\author{
Rosyida Permatasari ${ }^{1, a^{*}}$, Tholudin Mat Lazim ${ }^{2, b}$, Tono Sukarnoto $^{3, c}$ \\ and Supriyadi ${ }^{4, d}$ \\ 1, 2,4 Trisakti University, Kyai Tapa 1, Jakarta City, Indonesia \\ ${ }^{2}$ Universiti teknologi Malaysia, Skudai, Johor Bahru, Malaysia

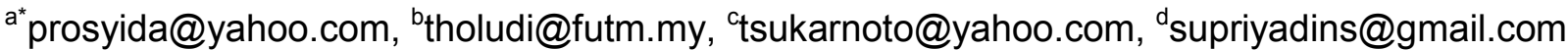

Keywords: gas-solid flow, PIV, CFD, fluidized bed combustion, fluidizing

\begin{abstract}
The fluidization behavior of a non-reactive gas-solid in the Atmospheric Bubbling Fluidized Bed Combustor (ABFBC) was studied. Experiments were conducted using laser based Particle Imaged Velocimetry (PIV) with B Geldart silica sand (diameter, $\varnothing=300-425 \mu \mathrm{m}$ ) in $2 \mathrm{~m}$ high cylindrical combustion chamber. The PIV was used to determine the particle velocity distribution in the combustion chamber. The experiments established the distribution of the sand along the height of the combustion chamber. Consequently, 3D CFD simulations were conducted using ANSYS FLUENT 13.0 software, of which their results were compared with the experimental counterpart. The comparison between the results of the developed CFD models and the experimental data showed very close agreement.
\end{abstract}

\section{Introduction}

Fluidization is a technique or a substantial reduction in the phenomenon of internal friction in the bed of solid particles with a counter force that the bed can flow like a fluid. Internal friction is caused by compression of the bed by gravity in many cases, but sometimes even by the buoyancy force when the particle density is lighter than the fluid density, by the magnetic force or by the centrifugal force. The counter forces, is a drag force of fluid flowing through the bed in the direction opposite the bed compression force. Direction of fluid flow is generally vertically upwards, but downwards to the floating particles. For centrifugal fluidization, it into the drum rotates. Sometimes counter-action is mechanical vibrations or sonic vibration [1].

The fluidization technology continuously developed such as the evaluation and validation of CFD models by measurements in industrial fluidized beds, the investigation of 3D effects in largescale fluidized beds and the development of measurement techniques for better process control [12]. Because, the general application of the gas/particle flow systems and fluidization in the industry are for an increase in efficiency and the improvement of fundamentally based on realistic simulations, accurate and detailed experimental data and design tools for such systems.

The measurement and numerical predictions of gas vortices formed by single eruptions in the freeboard of a BFB was investigated by S. Vun et al. [3]. Also B. Peng investigated the theoretical and numerical on the flow multiplicity phenomenon for gas-solids two-phase flows in CFB risers [4].

Meanwhile, Van Wachem et al. [5] investigated about the dynamic characteristics of the gassolids behavior at different superficial gas velocities, at different column diameters, and at different pressures. He also evaluated, namely the velocity of pressure and void age waves through the bed, the power of the low and high frequencies of the pressure and void age fluctuations, the reorientation of the gas-solids flow just above minimum fluidization and the effect of elevated pressure upon this reorientation, and the Kolmogorov entropy.

CFD simulations carried out for the predictions of flow pattern in bubble column reactors using 1D, 2D and 3D with $k-\varepsilon$ models. All the models showed good agreement with the experimental data for axial liquid velocity and the fractional gas hold-up profiles. However, for eddy diffusivity, only the 3D model predictions agree closely with the experimental data [6]. 
Measurement and numerical predictions of solid velocity were investigated in the freeboard of an ABFB. The experiments used a PIV measurement technique to visualize and measure the solid flow within the freeboard after a single bubble eruption. A computational study was carried out using Eulerian-Eulerian, kinetic theory of granular flow approach with $k$ - $\varepsilon$ model used to account for solid turbulence. Results from a three dimensional (3D) simulation of the experimental fluidized bed were compared with experimental velocity profiles of gas flow in the freeboard of the gas-solid fluidized bed after a bubble eruption.

\section{Computational Model}

The Eulerian granular model in ANSYS FLUENT was used to study the flow behavior in the model lab-scale ABFBC, in which the stress of the solid phase was described with the kinetic theory of granular flow; the drag coefficient correlation was corrected with consideration of particle clusters. The continuity and momentum equations for multiphase flow are obtained by time averaging process for each phase. These equations are analogous to the single phase Navier-Stoke equations but with some additional terms related to the interactions between the phases and there is also a pressure term for the solid phase.

Finite volume method was used to solve the set of governing equations. The governing equation can be summarised as follows.

The steady state equations of continuity for phase gas and solid with temporal and spatial gradients are given as:

$\frac{\partial}{\partial t}\left(\alpha_{g}+\rho_{g}\right)+\nabla \cdot\left(\alpha_{g} \rho_{g} \vec{v}_{g}\right)=0$

$\frac{\partial}{\partial \mathrm{t}}\left(\alpha_{\mathrm{s}}+\rho_{\mathrm{s}}\right)+\nabla \cdot\left(\alpha_{\mathrm{s}} \rho_{\mathrm{s}} \vec{v}_{\mathrm{s}}\right)=0$

The phase volume fractions satisfy the following condition: $\quad \alpha_{\mathrm{s}}+\alpha_{\mathrm{g}}=1$

The momentum balance for each phase given by the Navier-Stokes equation is modified to include an interphase momentum transfer term and a solid phase source term:

$$
\begin{aligned}
\frac{\partial}{\partial t}\left(\alpha_{g} \rho_{g} \vec{v}_{g}\right)+\nabla \cdot\left(\alpha_{g} \rho_{g} \vec{v}_{g} \vec{v}_{g}\right)=-\alpha_{g} \nabla \mathrm{p}+\nabla \cdot\left(\alpha_{g}\left(\overline{\overline{\tau_{g}^{m}}}+\overline{\overline{\tau_{g}^{R e}}}\right)\right) \\
+\alpha_{g} \rho_{g} \vec{g}+K_{s g}\left(\vec{v}_{s}-\vec{v}_{g}\right) \\
\frac{\partial}{\partial t}\left(\alpha_{s} \rho_{s} \vec{v}_{s}\right)+\nabla \cdot\left(\alpha_{s} \rho_{s} \vec{v}_{s} \vec{v}_{s}\right)=-\alpha_{s} \nabla \mathrm{p}+\nabla p_{s} \nabla \cdot\left(\alpha_{s}\left(\overline{\overline{\tau_{s}^{m}}}+\overline{\overline{\tau_{s}^{R e}}}\right)\right) \\
+\alpha_{s} \rho_{s} \vec{g}+K_{g s}\left(\vec{v}_{g}-\vec{v}_{s}\right)
\end{aligned}
$$

where the left side represents the temporal and spatial transport terms and the right side represents the various interacting forces. The interactions of each phase involve various momentum exchange mechanisms such as the drag, the lift and added mass force, etc. However, its effect of the other forces was ignored while its contribution of drag force was considered.

Analogous to the thermodynamic temperature for gases, the granular temperature $\Theta_{S}$ can be introduced as a measure of the particle velocity fluctuation, i.e.

$\Theta_{s}=\frac{1}{3} \vec{v}_{S}^{\prime 2}$

where: $\vec{v}_{s}^{\prime} \quad$ the solids fluctuating velocity.

Granular temperature is obtained by solving its transport equation that is:

$\frac{3}{2}\left\lfloor\frac{\partial}{\partial t}\left(\rho_{s} \alpha_{s} \Theta_{s}\right)+\nabla \cdot\left(\rho_{s} \alpha_{s} \Theta_{s}\right)\right]=\left(-p_{s} \overline{\bar{I}}+\overline{\overline{\tau_{s}}}\right): \nabla \vec{v}_{s}+\nabla \cdot\left(k_{\Theta_{\mathrm{s}}} \nabla \Theta_{s}\right)-\gamma_{\Theta_{\mathrm{s}}}+\Phi_{\mathrm{gs}}$ 
where:

$\left(-p_{s} \overline{\bar{I}}+\overline{\overline{\tau_{s}}}\right): \nabla \vec{v}_{s}$ is the generation of energy by the solid stress tensor because of the interaction between the normal and shear stress matrix with the mean velocity field, $k_{\Theta_{S}} \nabla \Theta_{s}$ is accounts for the transport of energy due to diffusion, $k_{\Theta_{S}}$ is diffusion coefficient and $\gamma_{\Theta_{S}}$ is dissipation of energy due to collision and its given as,

$$
\gamma_{\Theta_{\mathrm{s}}}=\frac{12\left(1-\mathrm{e}_{\mathrm{ss}}^{2}\right) \mathrm{g}_{0, \mathrm{ss}}}{\mathrm{d}_{\mathrm{s}} \sqrt{\pi}} \rho_{\mathrm{s}} \alpha_{\mathrm{s}}^{2} \Theta_{\mathrm{s}}^{3 / 2}
$$

$\Phi_{\mathrm{gs}}$ is the exchange of kinetic energy between the solid and the gas phases, given as,

$$
\Phi_{\mathrm{gs}}=-3 k_{\mathrm{gs}} \Theta_{\mathrm{s}}
$$

Granular temperature at the wall is

$$
q_{s}=\frac{\pi}{6} \sqrt{3} \Phi \frac{\alpha_{\mathrm{s}}}{\alpha_{\mathrm{s}, \max }} \rho_{\mathrm{s}} g_{0} \sqrt{\Theta_{s}} v_{t, w}^{2}-\frac{\pi}{4} \sqrt{3} \frac{\alpha_{\mathrm{s}}}{\alpha_{\mathrm{s}, \max }}\left(1-\mathrm{e}_{\mathrm{sw}}^{2}\right) \rho_{\mathrm{s}} g_{0} \Theta_{\mathrm{s}}^{3 / 2}
$$

The stress-strain tensor for each phase is given as,

$$
\begin{aligned}
& \overline{\overline{\tau_{s}^{m}}}=\left(\lambda_{s}-\frac{2}{3} \mu_{s}\right) \nabla \cdot \vec{v}_{s} \overline{\bar{I}}+\mu_{s}\left(\nabla \vec{v}_{s}+\nabla \vec{v}_{s}^{T}\right) \\
& \overline{\overline{\tau_{g}^{m}}}=\left(\lambda_{g}-\frac{2}{3} \mu_{g}\right) \nabla \cdot \vec{v}_{g} \overline{\bar{I}}+\mu_{g}\left(\nabla \vec{v}_{g}+\nabla \vec{v}_{g}^{T}\right)
\end{aligned}
$$

The Reynold stress tensor for each phase is given as,

$$
\begin{aligned}
& \overline{\overline{\tau_{s}^{R e}}}=-\frac{2}{3}\left(\rho k_{s}+\mu_{s, t} \nabla \cdot \vec{v}_{s}\right) \overline{\bar{I}}+\mu_{s, t}\left(\nabla \vec{v}_{s}+\nabla \vec{v}_{s}^{T}\right) \\
& \overline{\overline{\tau_{g}^{R e}}}=\frac{2}{3}\left(\rho k_{g}+\mu_{g, t} \nabla \cdot \vec{v}_{g}\right) \overline{\bar{I}}+\mu_{g, t}\left(\nabla \vec{v}_{g}+\nabla \vec{v}_{g}^{T}\right)
\end{aligned}
$$

The turbulent viscosities $\mu_{\mathrm{g}, \mathrm{t}}$ and $\mu_{\mathrm{s}, \mathrm{t}}$ are obtained by a modified standard $k-\varepsilon$ turbulence model include the interaction between the two phases. Its turbulent viscosity for each phase is given as:

$$
\begin{aligned}
& \mu_{g, t}=\rho_{g} C_{\mu} \frac{k_{g}^{2}}{\varepsilon_{g}} \\
& \mu_{s, t}=\rho_{s} C_{\mu} \frac{k_{s}^{2}}{\varepsilon_{s}}
\end{aligned}
$$

For the $k$ - $\varepsilon$ model, the $k$ equation for each phase is given as:

$$
\begin{gathered}
\quad \frac{\partial}{\partial t}\left(\alpha_{g} \rho_{g} k_{g}\right)+\nabla \cdot\left(\alpha_{g} \rho_{g} \vec{v}_{g} k_{g}\right)=\nabla \cdot\left(\alpha_{g} \frac{\mu_{g, t}}{\sigma_{k}} \nabla k_{g}\right)+\left(\alpha_{g} G_{g, k}-\alpha_{g} \rho_{g} \varepsilon_{g}\right)+K_{s g}\left(\begin{array}{c}
C_{s g} k_{s}- \\
C_{g s} k_{g}
\end{array}\right)- \\
K_{s g}\left(\vec{v}_{s}-\vec{v}_{g}\right) \cdot \frac{\mu_{s, t}}{\alpha_{s} \sigma_{s}} \nabla \alpha_{s}+K_{s g}\left(\vec{v}_{s}-\vec{v}_{g}\right) \cdot \frac{\mu_{g, t}}{\alpha_{g} \sigma_{g}} \nabla \alpha_{g} \\
\quad \frac{\partial}{\partial t}\left(\alpha_{s} \rho_{s} k_{s}\right)+\nabla \cdot\left(\alpha_{s} \rho_{s} \vec{v}_{s} k_{s}\right)=\nabla \cdot\left(\alpha_{s} \frac{\mu_{s, t}}{\sigma_{k}} \nabla k_{s}\right)+\left(\alpha_{s} G_{s, k}-\alpha_{s} \rho_{s} \varepsilon_{s}\right)+K_{g s}\left(C_{g s} k_{g}-C_{s} k_{s}\right)- \\
K_{g s}\left(\vec{v}_{g}-\vec{v}_{s}\right) \cdot \frac{\mu_{g, t}}{\alpha_{g} \sigma_{g}} \nabla \alpha_{g}+K_{g s}\left(\vec{v}_{g}-\vec{v}_{s}\right) \cdot \frac{\mu_{s, t}}{\alpha_{s} \sigma_{s}} \nabla \alpha_{s}
\end{gathered}
$$


The $\varepsilon$ equation for each phase is given as:

$$
\begin{aligned}
& \quad \frac{\partial}{\partial t}\left(\alpha_{g} \rho_{g} \varepsilon_{g}\right)+\nabla \cdot\left(\alpha_{g} \rho_{g} \vec{v}_{g} \varepsilon_{g}\right)=\nabla \cdot\left(\alpha_{g} \frac{\mu_{g, t}}{\sigma_{k}} \nabla \varepsilon_{g}\right)+\frac{\varepsilon_{g}}{k_{g}}\left(C_{1 \varepsilon} \alpha_{g} G_{g, k}-C_{2 \varepsilon} \alpha_{g} \rho_{g} \varepsilon_{g}+\right. \\
& \left.C_{3 \varepsilon}\left(K_{s g}\left(C_{s g} k_{s}-C_{g s} k_{g}\right)-K_{s g}\left(\vec{v}_{s}-\vec{v}_{g}\right) \cdot \frac{\mu_{s, t}}{\alpha_{s} \sigma_{s}} \nabla \alpha_{s}+K_{s g}\left(\vec{v}_{s}-\vec{v}_{g}\right) \cdot \frac{\mu_{g, t}}{\alpha_{g} \sigma_{g}} \nabla \alpha_{g}\right)\right) \\
& \quad \frac{\partial}{\partial t}\left(\alpha_{s} \rho_{s} \varepsilon_{s}\right)+\nabla \cdot\left(\alpha_{s} \rho_{s} \vec{v}_{s} \varepsilon_{s}\right)=\nabla \cdot\left(\alpha_{s} \frac{\mu_{s, t}}{\sigma_{k}} \nabla \varepsilon_{s}\right)+ \\
& \frac{\varepsilon_{s}}{k_{s}}\left(C_{1 \varepsilon} \alpha_{s} G_{s, k}-C_{2 \varepsilon} \alpha_{s} \rho_{s} \varepsilon_{s}+C_{3 \varepsilon}\left(K_{g s}\left(C_{g s} k_{g}-C_{s g} k_{s}\right)-K_{g s}\left(\vec{v}_{g}-\vec{v}_{s}\right) \cdot \frac{\mu_{g, t}}{\alpha_{g} \sigma_{g}} \nabla \alpha_{g}+K_{g s}\left(\vec{v}_{g}-\vec{v}_{s}\right) \cdot\right.\right. \\
& \left.\left.\frac{\mu_{s, t}}{\alpha_{s} \sigma_{s}} \nabla \alpha_{s}\right)\right)
\end{aligned}
$$

The last two terms on the right-hand side of $\mathrm{k}-\varepsilon$ equations are used to account for the effect of the solids phase on the gas phase turbulence or the gas phase on solids phase turbulence.

The interaction between gas and solids was expressed in the form of drag force, which is used to model the momentum exchange between gas phase and solids phase. Drag is caused by relative motion between phases. The drag coefficient is related to the flow regime and the properties of the two phases. The drag coefficient, Syamlal and O'Brien correlation, is commonly used to estimate for fluidized beds.

$$
K_{s g}=\frac{3 \alpha_{s} \alpha_{g} \rho_{g}}{4 v_{r, s}^{2} d_{s}}\left(0.63+\frac{4.8}{\sqrt{\mathrm{Re}_{\mathrm{s}}} / v_{r, s}}\right)^{2}\left(\frac{\mathrm{Re}_{\mathrm{s}}}{v_{r, s}}\right)\left|\vec{v}_{s}-\vec{v}_{g}\right|
$$

\begin{tabular}{|c|c|c|}
\hline Description & Value & Comment \\
\hline Particle density & $2500 \mathrm{~kg} / \mathrm{m}^{3}$ & Silica Sand \\
\hline Gas density & $1.2 \mathrm{~kg} / \mathrm{m}^{3}$ & Air \\
\hline Average particle diameter & $0,365 \mu \mathrm{m}$ & Uniform distribution \\
\hline Initial solid packing & 0.5 & Fixed value \\
\hline Superficial gas velocity & $5,76 \mathrm{~m} / \mathrm{s}$ & \\
\hline Bed height & $2 \mathrm{~m}$ & Fixed value \\
\hline Boundary condition & WALL & No-slip boundary condition \\
\hline Inlet boundary condition & VELOCITY & Superficial gas velocity \\
\hline Outlet boundary condition & OUTFLOW & Fully developed flow \\
\hline Time steps & $0.001 \mathrm{~s}$ & Fixed value \\
\hline Solids viscosity & Syamlal O’Brien & \\
\hline Iterative steps & 20 & Default in Fluent \\
\hline Convergence criteria & $10^{-3}$ & Default in Fluent \\
\hline
\end{tabular}

More information on the geometrical and operative conditions of the simulated fluidized bed can be seen in Table 1.

Table 1. Simulation Parameters

Fig.1 illustrates volume fraction of silica sand in central plane contours after different iterations. The illustrations involve three-dimensional fluidized bed, considering the plenum and the air distributor, and its dimensions correspond to the experimental facility $(0.144 \mathrm{~m} \times 2 \mathrm{~m})$. The results show that the calculation started to coverage after 1300 iterations, with the sand fill in the chamber to $2 \mathrm{~m}$ height from the chamber base. 

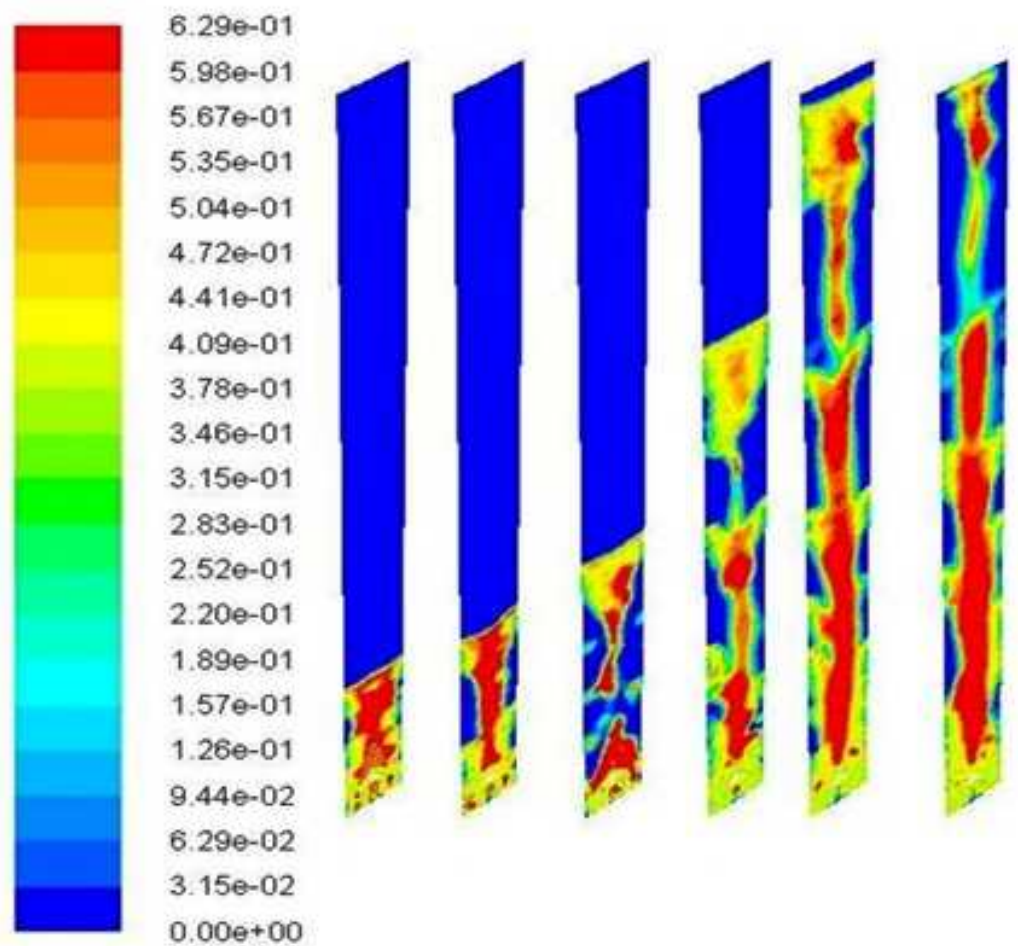

$(100)$

(200)

(300)

(800) (1300)

(1500)

Fig. 1. Central plane contours of volume fraction of silica sand (Phase 2) after different iterations

\section{Results Comparison}

This numerical simulation was carried out using ANSYS Fluent 13.0 to simulate the flow hydrodynamics inside the same geometry as the experimental model ABFBC. The inlet boundary condition was set as velocity inlet $5.76 \mathrm{~m} / \mathrm{s}$ and the outlet was set as pressure outlet at atmospheric pressure.

Fig. 2 shows the velocity distribution in the chamber at different heights. The velocities at the central axis were $0.1 \mathrm{~m} / \mathrm{s}$ for Heightl and between $0.75 \mathrm{~m} / \mathrm{s}$ to $1.2 \mathrm{~m} / \mathrm{s}$ for the other heights. The horizontal velocity distribution shows that the velocity was highest at the central axis and decreased slowly along the radius until $\mathrm{r}=60 \mathrm{~mm}(\mathrm{r} / \mathrm{R}=0.83)$. It then increased to a peak at $\mathrm{r}=67 \mathrm{~mm}$ $(\mathrm{r} / \mathrm{R}=0.93)$, and then dropping sharply to near zero at the wall. These profiles were similar with the results by Nan Zhang, et al. [8].

The simulated results from the developed model were validated using the experimental measurement, in terms of the velocity vector of the sand at 5 chosen heights, as shown in Fig. 2 and Fig. 3. In these comparisons, both measured experimental results and the simulated results were based on similar input variables.

At Height3 and Height4, the velocities are higher than Height1, as shown in Fig. 2. At these height, the radial distributions show similar trends between experimental and simulation results as shown in Fig. 3 and Fig. 4. The velocity from simulation is highest at the centre of the chamber but decreases towards the wall until about $r=48 \mathrm{~mm}$. Then it increased sharply to a maximum at $\mathrm{r}=64 \mathrm{~mm}$. Then it falls again to almost zero at the wall. The profile is similar to the experimental results, although the velocities are much lower. 


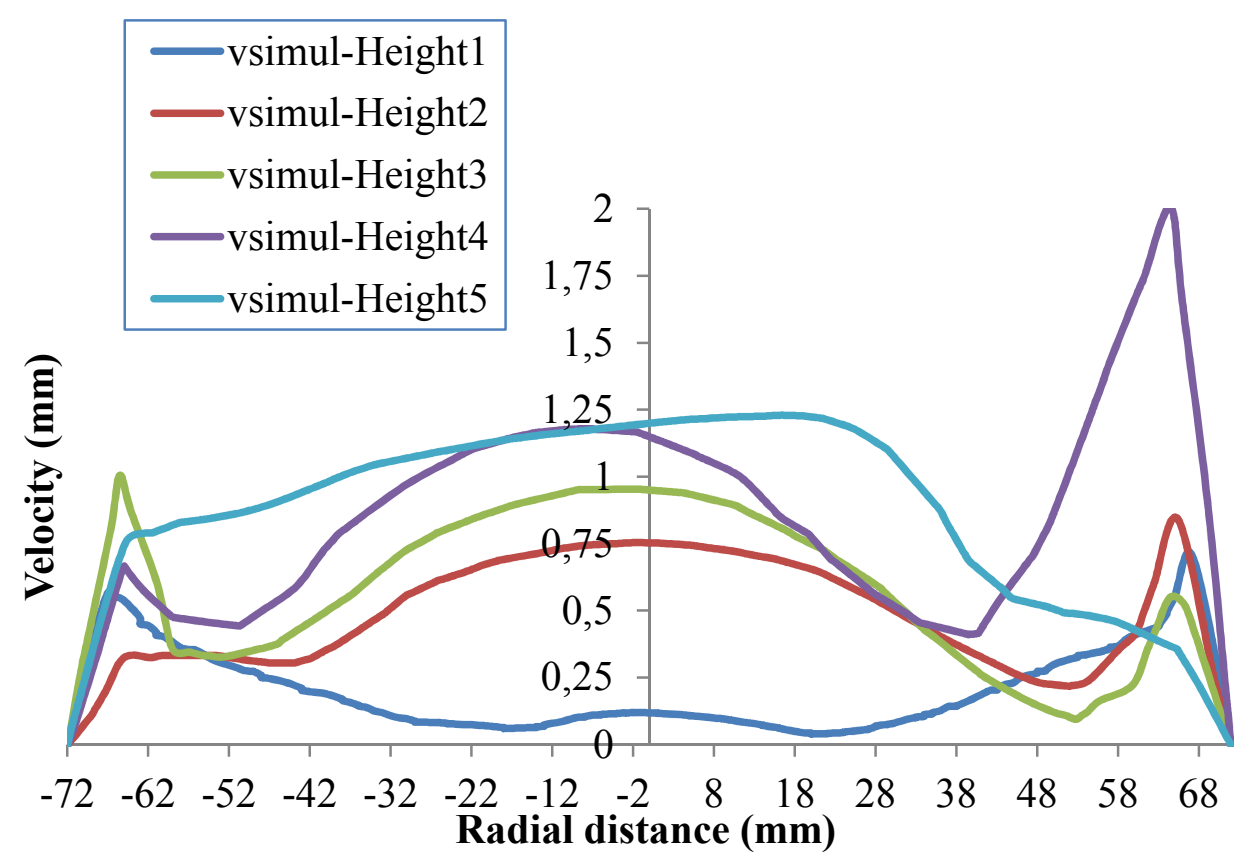

Fig. 2: NumericalVelocity Vectors of Silica Sand at $5.76 \mathrm{~m} / \mathrm{sAir}$ Velocity at Height1, Height2, Height3, Height4 and Height5

Fig. 3 and Fig. 4 show good agreement between experiment and simulation results Thus, it can be said that Height 3 and Height 4 are the ideal location to investigate velocity distribution using PIV measurements. This was so because of the sand concentration was much lower at these two heights, thus giving better image capture by the CCD camera. Furthermore, the thinner concentration allowed the sand to move more freely. The profiles are similar to those reported by Fan et al. [147].

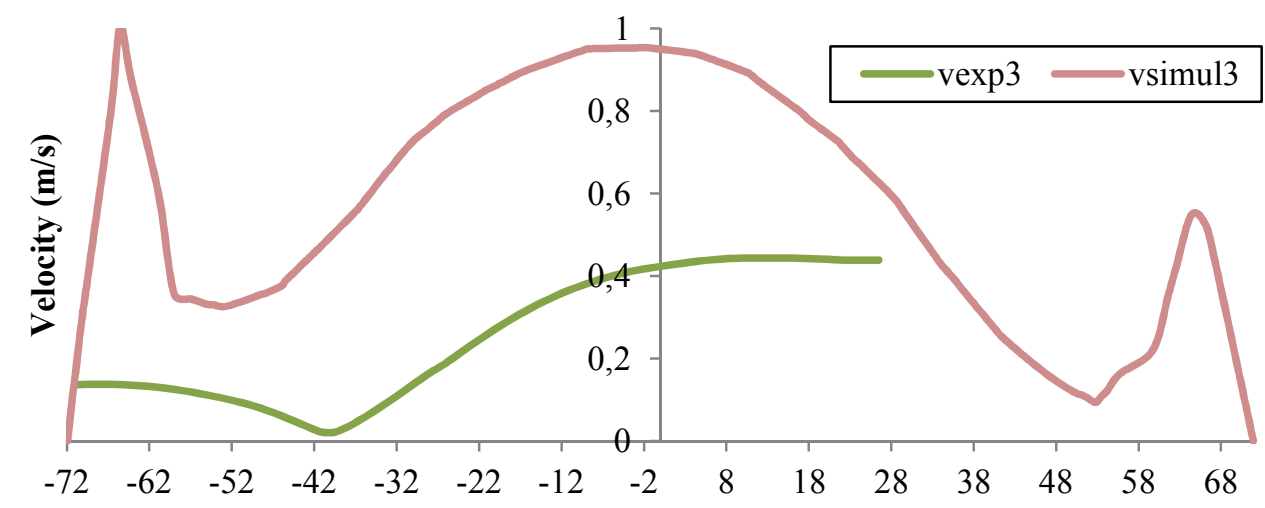

Distance - $\mathbf{x}(\mathbf{m m})$

Fig. 3: Experimental and Simulation Velocity of Silica Sand at 5.76m/s Air Velocity at Height3

Whenever there were similar trends in velocity distribution, their values still differ markedly. This can be explained by the fact that the sand granules were not spherical as was assumed in the simulation. Furthermore the sand granule sizes were actually very varied, with size variation from $300 \mu \mathrm{m}$ to $425 \mu \mathrm{m}$. Smaller size particles move up higher in the freeboard whilst bigger one tended to be at the bottom. 


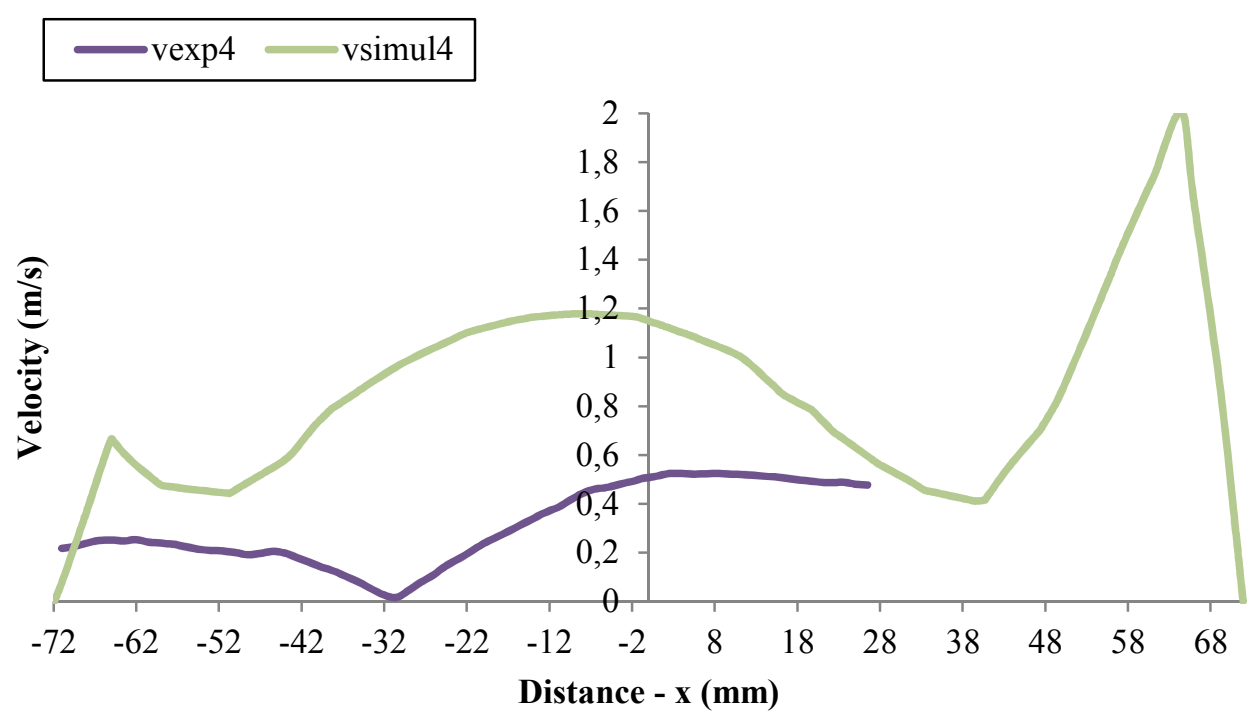

Fig. 4: Experimental and Simulation Velocity of Silica Sand at $5.76 \mathrm{~m} / \mathrm{s}$ Air Velocity at Height4

\section{Conclusion}

Two major kinds of information have been analyzed from this work, such as at all cross-sections, velocities of particles were measured which in the core region the velocities were higher than near the wall region and the comparison between the developed numerical model and experimental data on velocity profiles at axial distance showed very close agreement.

\section{Acknowledgment}

This presentation work has been partially funded by the Ministry of Higher Education of Indonesia. Their supports are greatly appreciated.

\section{References}

[1] Horio, M. Fluidization Science, Its Development and Future, Particuology. 8 (2010) 514-524.

[2] Werther, J. Fluidization Technology Development - the Industry/Academia Collaboration Issue, Powder Technology. 113 (2000) 230-241.

[3] Vun, S., Naser, J., Witt, P. J. and Yang, W. Measurements and Numerical Predictions of Gas Vortices Formed by Single Bubble Eruptions in the Freeboard of a Fluidised Bed, Chemical Engineering Science. 65 (2010) 5808-5820.

[4] Peng, B., Zhang, C. and Zhu, J. Theoretical and Numerical Studies on the Flow Multiplicity Phenomenon for Gas-Solids Two-Phase Flows in Cfb Risers, International Journal of Multiphase Flow. 37 (2011) 660-670.

[5] Van Wachem, B. G. M., Schouten, J. C., Krishna, R. and van den Bleek, C. M. Validation of the Eulerian Simulated Dynamic Behaviour of Gas-Solid Fluidised Beds, Chemical Engineering Science. 54 (1999) 2141-2149.

[6] Ekambara, K., Dhotre, M. T. and Joshi, J. B. Cfd Simulations of Bubble Column Reactors: 1d, 2d and 3d Approach, Chemical Engineering Science. 60 (2005) 6733-6746.

[7] Zhang, N., Lu, B., Wang, W. and Li, J. 3d Cfd Simulation of Hydrodynamics of a 50\&\#Xa0; Mwe Circulating Fluidized Bed Boiler, Chemical Engineering Journal. 162 (2010) 821828.

[8] Fan, X., Yang, Z., Parker, D. J. and Armstrong, B. Prediction of Bubble Behaviour in Fluidised Beds Based on Solid Motion and Flow Structure, Chemical Engineering Journal. 140 (2008) 358-369. 